Canad. Math. Bull. Vol. 62 (2), 2019 pp. 383-392

http://dx.doi.org/10.4153/CMB-2018-032-6

(C) Canadian Mathematical Society 2018

\title{
Generating Curves of Minimal Ruled Real Hypersurfaces in a Nonflat Complex Space Form
}

\author{
Sadahiro Maeda, Hiromasa Tanabe, and Seiichi Udagawa
}

Abstract. We first provide a necessary and sufficient condition for a ruled real hypersurface in a nonflat complex space form to have constant mean curvature in terms of integral curves of the characteristic vector field on it. This yields a characterization of minimal ruled real hypersurfaces by circles. We next characterize the homogeneous minimal ruled real hypersurface in a complex hyperbolic space by using the notion of strong congruency of curves.

\section{Introduction}

It is possible in some cases to know properties of a submanifold by examining the behavior of some curves on the submanifold. In this paper, we study ruled real hypersurfaces $M$ in a nonflat complex space form $\widetilde{M}_{n}(c), n \geq 2$, in terms of their generating curves.

An $n$-dimensional nonflat complex space form $\widetilde{M}_{n}(c)$ of constant holomorphic sectional curvature $c$ is a complex $n$-dimensional complete and simply connected Kähler manifold, all of whose holomorphic sectional curvatures take the same value $c(\neq 0)$. It is known that this space $\widetilde{M}_{n}(c)$ is holomorphically isometric to a complex projective space $\mathbb{C} P^{n}(c)$ or a complex hyperbolic space $\mathbb{C} H^{n}(c)$ according as $c>0$ or $c<0$. A ruled real hypersurface $M^{2 n-1}$ in $\widetilde{M}_{n}(c)$ is a real hypersurface having a one-codimensional foliation whose leaves are totally geodesic complex hyperplanes $\widetilde{M}_{n-1}(c)$ (for a precise definition, see Section 3). Such a hypersurface can be constructed as follows: given an arbitrary regular smooth curve $\gamma: I \rightarrow \widetilde{M}_{n}(c)$ defined on an open interval $I(\subset \mathbb{R})$, we attach a totally geodesic complex hyperplane $M_{s} \cong \widetilde{M}_{n-1}(c)$ to each point $\gamma(s)(s \in I)$ in such a way that the plane $M_{s}$ is orthogonal not only to $\dot{\gamma}(s)$ but to $J \dot{\gamma}(s)$. Then the union $M_{\gamma}:=\bigcup_{s \in I} M_{s}$ is a ruled real hypersurface in $\widetilde{M}_{n}(c)$. We call this $M_{\gamma}$ and the curve $\gamma$ a ruled real hypersurface associated with $\gamma$ and a generating curve of $M_{\gamma}$, respectively. Note that every integral curve of the characteristic vector field on a ruled real hypersurface is a generating curve of it.

In the class of ruled real hypersurfaces, which is an abundant class as one can see from the above construction, we have important examples that are minimal. Lohnherr and Reckziegel [6] characterized minimal ruled real hypersurfaces in a nonflat

Received by the editors May 21, 2018; revised July 10, 2018.

Published electronically December 1, 2018.

AMS subject classification: 53B25, 53C40.

Keywords: nonflat complex space form, minimal ruled real hypersurface, constant mean curvature, integral curves of the characteristic vector field. 
complex space form $\widetilde{M}_{n}(c), n \geq 2$, by the property that their generating curves are totally real circles, that is, circles in some totally real totally geodesic real twodimensional submanifold $\mathbb{R} M^{2}(c / 4)$, in $\widetilde{M}_{n}(c)$ (i.e., $\mathbb{R} P^{2}(c / 4)$ or $\mathbb{R} H^{2}(c / 4)$ ), and they classified such hypersurfaces in three families according to the shapes of generating curves. Also, Adachi, Bao, and Maeda [1] studied congruency of minimal ruled real hypersurfaces in $\widetilde{M}_{n}(c)$ with respect to the action of its isometry group. Among others, the homogeneous minimal ruled real hypersurface, the so-called Lohnherr hypersurface, in $\mathbb{C H}(c)$ is more significant, because it is the only example of a homogeneous real hypersurface that is minimal in $\mathbb{C} H^{n}(c)$ [4]. Here, a homogeneous real hypersurface in $\widetilde{M}_{n}(c)$ is an orbit of some subgroup of the full isometry group $\mathrm{I}\left(\widetilde{M}_{n}(c)\right)$.

Inspired by these facts, we establish the following theorem.

Theorem $1.1 \quad$ Let $M$ be a ruled real hypersurface in a nonflat complex space form $\widetilde{M}_{n}(c), n \geq 2$. Then $M$ has constant mean curvature, i.e., Trace $A$ is constant on $M$, where $A$ is the shape operator of $M$ in $\widetilde{M}_{n}(c)$, if and only if every integral curve of the characteristic vector field on $M$ is a circle in $\widetilde{M}_{n}(c)$.

Recently, M. Domínguez-Vázquez and O. Pérez-Barral [5] proved that there is no ruled real hypersurface with nonzero constant mean curvature in nonflat complex space forms. Hence, combining the result in [5] with [6, Theorem 4] and our Theorem 1.1, we have the following.

Corollary $1.2 \quad$ For a ruled real hypersurface $M$ in a nonflat complex space form $\widetilde{M}_{n}(c), n \geq 2$, the following four conditions are mutually equivalent.

(i) $M$ is minimal in $\widetilde{M}_{n}(c)$.

(ii) $M$ has constant mean curvature.

(iii) Every integral curve of the characteristic vector field on $M$ is a circle in $\widetilde{M}_{n}(c)$.

(iv) An, hence every, integral curve of the characteristic vector field on $M$ is a totally real circle in $\widetilde{M}_{n}(c)$.

In order to describe Theorem 1.3, we explain the congruency of curves. Let $\mathrm{I}(\widetilde{M})$ be a full isometry group of a Riemannian manifold $\widetilde{M}$. We say that smooth curves $\gamma_{1}=\gamma_{1}(s)$ and $\gamma_{2}=\gamma_{2}(s)$ in $\widetilde{M}$ are congruent in the usual sense if there exist $\varphi \in \mathrm{I}(\widetilde{M})$ and a constant $s_{0} \in \mathbb{R}$ with $\gamma_{2}(s)=\left(\varphi \circ \gamma_{1}\right)\left(s+s_{0}\right)$ for all $s$. If there exists $\varphi \in \mathrm{I}(\widetilde{M})$ with $\gamma_{2}(s)=\left(\varphi \circ \gamma_{1}\right)(s)$ for all $s$, we say that they are strongly congruent. We can find that a Riemannian manifold $\widetilde{M}$ is congruent to either a Euclidean space or a Riemannian symmetric space of rank one if and only if all geodesics on $\widetilde{M}$ are strongly congruent with each other by isometries of $\widetilde{M}$.

Let $M$ be an arbitrary Hopf hypersurface (for definition, see Section 3) in a nonflat complex space form $\widetilde{M}_{n}(c), n \geq 2$. It is easy to see that all integral curves of the characteristic vector field on $M$ are strongly congruent with each other by holomorphic isometries of $\widetilde{M}_{n}(c)$ and that they are circles of the same curvature that lie in a totally geodesic $\widetilde{M}_{1}(c)$ in $\widetilde{M}_{n}(c)$. For a ruled real hypersurface, we have the following. 
Theorem 1.3 Let $M$ be a ruled real hypersurface in a nonflat complex space form $\widetilde{M}_{n}(c), n \geq 2$. If all integral curves of the characteristic vector field on $M$ are strongly congruent with each other by holomorphic isometries of $\widetilde{M}_{n}(c)$, then the ambient space $\widetilde{M}_{n}(c)$ must be a complex hyperbolic space $\mathbb{C} H^{n}(c)$ and the ruled real hypersurface $M$ is homogeneous and minimal in $\mathbb{C} H^{n}(c)$.

As an application, we have the following.

Corollary 1.4 Let $M$ be a ruled real hypersurface in a nonflat complex space form $\widetilde{M}_{n}(c), n \geq 2$. If all integral curves of the characteristic vector field on $M$ are circles of the same curvature in $\widetilde{M}_{n}(c)$, then the ambient space $\widetilde{M}_{n}(c)$ must be a complex hyperbolic space $\mathbb{C H}^{n}(c)$ and the ruled real hypersurface $M$ is homogeneous and minimal in $\mathbb{C} H^{n}(c)$.

\section{Curves in a Nonflat Complex Space Form}

First, we shall make a survey of the real curve theory in a nonflat complex space form $\widetilde{M}_{n}(c), n \geq 2$. Let $\gamma: I \rightarrow \widetilde{M}_{n}(c)$ be a smooth real curve parametrized by its arclength $s$ defined on an open interval $I(\subset \mathbb{R})$ and put $V_{1}:=\dot{\gamma}$. We then call the function $\kappa_{1}(s):=\left\|\widetilde{\nabla}_{\dot{\gamma}} V_{1}(s)\right\|$ the first curvature of $\gamma$, where $\widetilde{\nabla}$ is the Riemannian connection of $\widetilde{M}_{n}(c)$. A point $\gamma\left(s_{0}\right)\left(s_{0} \in I\right)$ with $\kappa_{1}\left(s_{0}\right)=0$ is said to be an inflection point of $\gamma$. For the curve $\gamma$ having no inflection points, one can define a unit vector field $V_{2}$ along $\gamma$ by $V_{2}:=\left(1 / \kappa_{1}\right) \widetilde{\nabla}_{\dot{\gamma}} V_{1}$. Then one finds

$$
\widetilde{\nabla}_{\dot{\gamma}} V_{1}=\kappa_{1} V_{2}, \quad \widetilde{\nabla}_{\dot{\gamma}} V_{2}=-\kappa_{1} V_{1}+W
$$

with some vector field $W$ along $\gamma$ that is orthogonal to $V_{1}$ and $V_{2}$. If $\kappa_{2}:=\|W\|$ vanishes at $s_{0} \in I$, we say $\gamma$ is of proper order 2 at $\gamma\left(s_{0}\right)$. If $\kappa_{2}=\|W\|$ does not vanish on $I$, we put $V_{3}:=\left(1 / \kappa_{2}\right) W$ and consider $\widetilde{\nabla}_{\dot{\gamma}} V_{3}$. Generally, a curve $\gamma$ is said to be a Frenet curve of proper order $d(2 \leq d \leq 2 n)$ if there exist an orthonormal system $\left\{V_{1}=\dot{\gamma}, V_{2}, \ldots, V_{d}\right\}$ of vector fields along $\gamma$ and positive smooth functions $\kappa_{1}(s), \ldots, \kappa_{d-1}(s)$ such that they satisfy the following

$$
\widetilde{\nabla}_{\dot{\gamma}} V_{j}(s)=-\kappa_{j-1}(s) V_{j-1}(s)+\kappa_{j}(s) V_{j+1}(s), \quad 1 \leq j \leq d .
$$

Here, $\kappa_{0} V_{0}$ and $\kappa_{d} V_{d+1}$ are null vector fields along $\gamma$. The functions $\kappa_{1}, \ldots, \kappa_{d-1}$ and the orthonormal frame $\left\{V_{1}, V_{2}, \ldots, V_{d}\right\}$ are called the curvatures and the Frenet frame of the curve $\gamma$, respectively. Equation (2.1) is known as the Frenet formula.

For the Frenet frame $\left\{V_{1}, \ldots, V_{d}\right\}$ of a Frenet curve $\gamma$ of proper order $d$ in $\widetilde{M}_{n}(c)$, we set $\tau_{i j}(s):=g\left(V_{i}(s), J V_{j}(s)\right)$ with $1 \leq i<j \leq d$ and call them the holomorphic torsions of $\gamma$, where $g$ and $J$ are the Riemannian metric and the Kähler structure of $\widetilde{M}_{n}(c)$, respectively. The notion of holomorphic torsions plays a distinguished role in the study of Frenet curves in $\widetilde{M}_{n}(c)$. Recall the definition of "strong congruence" of curves. Then the congruence theorem for Frenet curves in $\widetilde{M}_{n}(c)$ can be described in terms of curvatures and holomorphic torsions, as follows.

Theorem $A$ ([7]) Let $\gamma=\gamma(s)$ and $\sigma=\sigma(s)$ be two Frenet curves of proper orders $d$ and $d^{\prime}$ in a complex space form $\widetilde{M}_{n}(c)$, respectively. Let $\kappa_{1}^{\gamma}, \ldots, \kappa_{d-1}^{\gamma}$ (resp. 
$\left.\kappa_{1}^{\sigma}, \ldots, \kappa_{d^{\prime}-1}^{\sigma}\right)$ be the curvatures of $\gamma$ (resp. $\sigma$ ), and $\tau_{i j}^{\gamma}$ (resp. $\tau_{k l}^{\sigma}$ ) be holomorphic torsions of $\gamma$ (resp. $\sigma$ ). Then the two curves $\gamma$ and $\sigma$ are strongly congruent by a holomorphic isometry of $\widetilde{M}_{n}(c)$ if and only if $d=d^{\prime}, \kappa_{i}^{\gamma}(s)=\kappa_{i}^{\sigma}(s)$ for $1 \leq i \leq d-1$ and $\tau_{i j}^{\gamma}(0)=\tau_{i j}^{\sigma}(0)$ for $1 \leq i \leq d$.

A Frenet curve of proper order 2 with constant first curvature $\kappa_{1}=k(>0)$, that is to say, a curve that satisfies $\widetilde{\nabla}_{\dot{\gamma}} \dot{\gamma}=k V_{2}$ and $\widetilde{\nabla}_{\dot{\gamma}} V_{2}=-k \dot{\gamma}$, is called a circle of curvature $k$. We regard a geodesic as a circle of null curvature. For every fixed point $p \in \widetilde{M}_{n}(c)$, each pair of orthonormal vectors $X, Y$ of $T_{p} \widetilde{M}_{n}(c)$, and an arbitrary positive constant $k$, there exists locally a unique circle of curvature $k$ satisfying the initial condition that $\gamma(0)=p, \dot{\gamma}(0)=X$, and $\left(\widetilde{\nabla}_{\dot{\gamma}} \dot{\gamma}\right)(0)=k Y$.

We find that the holomorphic torsion $\tau_{12}$ of the circle $\gamma$ with positive curvature $k$ is constant along $\gamma$ as follows:

$$
\widetilde{\nabla}_{\dot{\gamma}}\left(g\left(V_{1}, J V_{2}\right)\right)=g\left(\widetilde{\nabla}_{\dot{\gamma}} \dot{\gamma}, J V_{2}\right)+g\left(\dot{\gamma}, J \widetilde{\nabla}_{\dot{\gamma}} V_{2}\right)=k g\left(V_{2}, J V_{2}\right)-k g(\dot{\gamma}, J \dot{\gamma})=0 .
$$

A circle of positive curvature in $\widetilde{M}_{n}(c)$ is said to be totally real if its holomorphic torsion $\tau_{12}$ vanishes. Such a circle lies on a totally real totally geodesic surface $\mathbb{R} M^{2}(c / 4)$ of $\widetilde{M}_{n}(c)$ (i.e., $\mathbb{R} P^{2}(c / 4)$ or $\mathbb{R} H^{2}(c / 4)$ ).

\section{Real Hypersurfaces in a Nonflat Complex Space form}

In this section we summarize some fundamental notions on real hypersurfaces in a nonflat complex space form. Let $\widetilde{M}_{n}(c)$ be an $n(\geq 2)$-dimensional nonflat complex space form furnished with standard Riemannian metric $g$ and the canonical Kähler complex structure $J$, and let $M^{2 n-1}$ be a real hypersurface of $\widetilde{M}_{n}(c)$ through an isometric immersion. Denote by $\mathcal{N}$ a unit normal local vector field on $M$ and by $A$ the shape operator of $M$ in $\widetilde{M}_{n}(c)$. We also denote by the same notation $g$ the induced Riemannian metric on $M$. Then the Riemannian connections $\widetilde{\nabla}$ of $\widetilde{M}_{n}(c)$ and $\nabla$ of $M$ are related by Gauss and Weingarten formulas

$$
\widetilde{\nabla}_{X} Y=\nabla_{X} Y+g(A X, Y) \mathcal{N}, \quad \widetilde{\nabla}_{X} \mathcal{N}=-A X
$$

for vector fields $X$ and $Y$ tangent to $M$.

An odd-dimensional manifold $M^{2 n-1}$ is said to have an almost contact structure if it admits a $(1,1)$-tensor field $\phi$ and a vector field $\xi$ with dual 1-form $\eta$, i.e., $\eta(\xi)=1$, such that $\phi^{2}=-I+\eta \otimes \xi$, where $I$ denotes the identity map of the tangent bundle $T M$ of $M$. The vector field $\xi$ is called the characteristic or Reeb vector field and the 1-form $\eta$ is called the contact form on $M$. The structure satisfies $\phi \xi=0$ and $\eta \circ \phi=$ 0 . In addition, if there exists a Riemannian metric $g$ on $M$ satisfying $g(\phi X, \phi Y)=$ $g(X, Y)-\eta(X) \eta(Y)$, then we say that $M$ has an almost contact metric structure.

For any real hypersurface $M$ isometrically immersed into $\widetilde{M}_{n}(c), n \geq 2$, an almost contact metric structure $(\phi, \xi, \eta, g)$ on $M$ is naturally induced from the Kähler structure $J$ of the ambient space $\widetilde{M}_{n}(c)$ as

$$
\xi:=-J \mathcal{N}, \quad \eta(X):=g(\xi, X)=g(J X, \mathcal{N}), \quad \text { and } \quad \phi X:=J X-\eta(X) \mathcal{N} .
$$


Moreover, we have the following equations.

$$
\begin{aligned}
\nabla_{X} \xi & =\phi A X, \\
\left(\nabla_{X} \phi\right) Y & =\eta(Y) A X-g(A X, Y) \xi \\
\left(\nabla_{X} A\right) Y-\left(\nabla_{Y} A\right) X & =(c / 4)\{\eta(X) \phi Y-\eta(Y) \phi X-2 g(\phi X, Y) \xi\} .
\end{aligned}
$$

The last one is known as Codazzi's equation.

We call an eigenvalue and an eigenvector of the shape operator of $A$ a principal curvature and a principal curvature vector of $M$ in $\widetilde{M}_{n}(c)$, respectively. If the characteristic vector $\xi$ is a principal curvature vector at each point of $M$, the hypersurface $M$ is said to be a Hopf hypersurface. Every homogeneous real hypersurface in $\mathbb{C} P^{n}(c)$ is a Hopf hypersurface $[9,10]$, whereas there exist many non-Hopf homogeneous real hypersurfaces in $\mathbb{C} H^{n}(c)$ [4]. Ruled real hypersurfaces are typical examples of nonHopf hypersurfaces in $\widetilde{M}_{n}(c)$.

Next, we give a precise definition of ruled real hypersurfaces in a nonflat complex space form and recall some basic results. Let $M$ be a real hypersurface of $\widetilde{M}_{n}(c)$, $n \geq 2$. We define the holomorphic distribution $T^{0} M$ on $M$ by

$$
T^{0} M:=\{X \in T M \mid \eta(X)=0\},
$$

where $\eta$ is the contact form on $M$. A real hypersurface $M$ is said to be ruled if the distribution $T^{0} M$ is integrable and each of its leaves is locally congruent to a totally geodesic complex hypersurface $\widetilde{M}_{n-1}(c)$ of the ambient space. The construction of ruled real hypersurfaces stated in the introduction implies that a ruled real hypersurface may have singularities in general. Hence, we must omit such points.

For any real hypersurface $M$ in $\widetilde{M}_{n}(c)$, we define two functions $\mu, v: M \rightarrow \mathbb{R}$ by

$$
\mu:=g(A \xi, \xi), \quad v:=\|A \xi-\mu \xi\|,
$$

where $A$ denotes the shape operator of $M$ in $\widetilde{M}_{n}(c)$. We also define the subsets $M_{0}$, $M_{*}$ of $M$ by

$$
M_{0}:=\{p \in M \mid v(p)=0\}, \quad M_{*}:=\{p \in M \mid v(p)>0\} .
$$

Then the following is well known.

Lemma 3.1 ( [8]) A real hypersurface $M$ in $\widetilde{M}_{n}(c)$ is ruled if and only if the following holds. The set $M_{*}$ is an open dense subset of $M$ and there exists a unit vector field $U$ on $M_{*}$ that is orthogonal to $\xi$ and satisfies

$$
A \xi=\mu \xi+v U, \quad A U=v \xi, \quad \text { and } \quad A X=0
$$

for any tangent vector $X$ orthogonal to both $\xi$ and $U$.

One can easily see from (3.4) that a ruled real hypersurface $M$ has three distinct principal curvatures, $(\mu / 2) \pm \sqrt{\left(\mu^{2} / 4\right)+v^{2}}$ and 0 , on $M_{*}$. A ruled real hypersurface $M$ is minimal if and only if the function $\mu$ vanishes on $M$. Moreover, when the ambient space is a complex hyperbolic space $\mathbb{C} H^{n}(c)$, we have the following. 
Theorem $B([1,4,6])$ For a ruled real hypersurface $M$ in $\mathbb{C} H^{n}(c), n \geq 2$, the following four conditions are mutually equivalent.

(i) $M$ is homogeneous in $\mathbb{C} H^{n}(c)$.

(ii) $M$ has constant principal curvatures.

(iii) The functions $\mu$ and $v$ satisfy $\mu \equiv 0$ and $v \equiv \sqrt{|c|} / 2$.

(iv) There exists a generating curve $\gamma$ of $M$ such that the curve $\gamma$ is a totally real circle of curvature $\sqrt{|c|} / 2$ in $\mathbb{C} H^{n}(c)$.

\section{Proof of Theorems}

The proof will require some relations.

Lemma 4.1 Let $M$ be a ruled real hypersurface of a nonflat complex space form $\widetilde{M}_{n}(c), n \geq 2$. Denote by $\nabla$ the Riemannian connection on $M$. Then on the open dense subset $M_{*}=\{p \in M \mid v(p)>0\}$ we have the following.

$$
\begin{gathered}
\nabla_{\xi} \xi=v \phi U, \\
\nabla_{\xi}(\phi U)=-v \xi+\phi \nabla_{\xi} U, \\
X \mu=\xi(v g(X, U))+\mu v g(X, \phi U)-v g\left(\nabla_{\xi} X, U\right) \quad \text { for } X \in T^{0} M, \\
X v=\left\{v^{2}+(c / 4)\right\} g(X, \phi U) \quad \text { for } X \in T^{0} M, \\
(\phi U) \mu=\mu \nu-v g\left(\phi \nabla_{\xi} U, U\right), \\
U \mu=\xi v .
\end{gathered}
$$

Proof Relations (4.1) and (4.2) immediately follow from (3.1), (3.2), and (3.4). Next, by Codazzi's equation (3.3) we have

$$
\left(\nabla_{X} A\right) \xi-\left(\nabla_{\xi} A\right) X=-(c / 4) \phi X \quad \text { for } X \in T^{0} M .
$$

On the other hand, we decompose a vector $X \in T^{0} M$ into $X=g(X, U) U+X^{\prime}$ with $X^{\prime} \in T^{0} M, X^{\prime} \perp U$. Using (3.4) we find for $X \in T^{0} M$

$$
A X=v g(X, U) \xi
$$

so that

$$
\nabla_{X} \xi=\phi A X=0 .
$$

Furthermore, writing the vector $\nabla_{\xi} X$ for $X \in T^{0} M$ as

$$
\nabla \xi X=g\left(\nabla_{\xi} X, \xi\right) \xi+g\left(\nabla_{\xi} X, U\right) U+Y
$$

with some $Y \in T^{0} M, Y \perp U$, we observe that

$$
\begin{aligned}
A \nabla_{\xi} X & =\mu g\left(\nabla_{\xi} X, \xi\right) \xi-v g\left(X, \nabla_{\xi} \xi\right) U+v g\left(\nabla_{\xi} X, U\right) \xi \\
& =\mu g\left(\nabla_{\xi} X, \xi\right) \xi-v^{2} g(X, \phi U) U+v g\left(\nabla_{\xi} X, U\right) \xi
\end{aligned}
$$


by use of (3.4) and (4.1). We then see from (3.4), (4.1), (4.8), (4.9), and (4.10) that

$$
\begin{aligned}
\left(\nabla_{X} A\right) \xi- & \left(\nabla_{\xi} A\right) X \\
= & \nabla_{X}(A \xi)-A \nabla_{X} \xi-\nabla_{\xi}(A X)+A \nabla_{\xi} X \\
= & \nabla_{X}(\mu \xi+v U)-\nabla_{\xi}(v g(X, U) \xi) \\
& \quad+\mu g\left(\nabla_{\xi} X, \xi\right) \xi-v^{2} g(X, \phi U) U+v g\left(\nabla_{\xi} X, U\right) \xi \\
= & (X \mu) \xi+(X v) U+v \nabla_{X} U-\xi(v g(X, U)) \xi-v^{2} g(X, U) \phi U \\
& \quad-\mu v g(X, \phi U) \xi-v^{2} g(X, \phi U) U+v g\left(\nabla_{\xi} X, U\right) \xi .
\end{aligned}
$$

Note that $v \nabla_{X} U$ is orthogonal to both $\xi$ and $U$. Then, taking the inner product of $\left(\nabla_{X} A\right) \xi-\left(\nabla_{\xi} A\right) X$ with $\xi$ and $U$, one can get relations (4.3) and (4.4) in our lemma from (4.7) and (4.11). The last two relations, (4.5) and (4.6), can be obtained from (4.3) by putting $X=\phi U, U$.

We shall investigate the extrinsic shape of integral curves of the characteristic vector field $\xi$ on $M$. We argue on the open dense subset $M_{*}=\{p \in M \mid v(p)>0\}$ of $M$. Let $\gamma$ be an integral curve of $\xi$ that is contained in $M_{*}$. Since the unit vector field $U$ can be defined on $M_{*}$, it follows from (3.4), and (4.1) and the Gauss formula that

$$
\widetilde{\nabla}_{\dot{\gamma}} \dot{\gamma}=\nu \phi U+\mu \mathcal{N}
$$

so that the first curvature $\kappa_{1}$ of $\gamma$, regarding $\gamma$ as a curve in $\widetilde{M}_{n}(c)$, is given by

$$
\kappa_{1}(s)=\sqrt{v(\gamma(s))^{2}+\mu(\gamma(s))^{2}} .
$$

As $v>0$ on $M_{*}$, the curve has no inflection points and one can define a unit vector $V_{2}$ along $\gamma$ by

$$
V_{2}(s):=\left(1 / \kappa_{1}(s)\right)\left\{v(\gamma(s)) \phi U_{\gamma(s)}+\mu(\gamma(s)) \mathcal{N}_{\gamma(s)}\right\}
$$

We thus find

$$
\begin{aligned}
\tau_{12}(s) & =g\left(\dot{\gamma}(s), J V_{2}(s)\right) \\
& =-\left(1 / \kappa_{1}(s)\right) g\left(J \xi_{\gamma(s)}, v(\gamma(s)) \phi U_{\gamma(s)}+\mu(\gamma(s)) \mathcal{N}_{\gamma(s)}\right) \\
& =-\mu(\gamma(s)) / \kappa_{1}(s)
\end{aligned}
$$

Now we demonstrate Theorem 1.1.

Proof of Theorem 1.1 Let $M$ be a ruled real hypersurface in a nonflat complex space form $\widetilde{M}_{n}(c), n \geq 2$, and let $\gamma$ be an integral curve of the characteristic vector field $\xi$.

We first assume that $M$ has constant mean curvature. Let us consider the open dense subset $M_{*}$. It then follows from the representation (3.4) of the shape operator $A$ on $M_{*}$ that $\mu$ is a constant function on $M_{*}$ (hence on the whole of $M$ ). Thus relation (4.6) gives $\xi v=0$, that is, the function $v$ is constant along each integral curve $\gamma$ of $\xi$. From (4.13) we see the first curvature $\kappa_{1}$ is constant along $\gamma$. Then, by using Gauss 
and Weingarten formulas and (3.4), (4.2), and (4.14) we have

$$
\begin{aligned}
\widetilde{\nabla}_{\dot{\gamma}} V_{2} & =\left(v / \kappa_{1}\right) \widetilde{\nabla}_{\dot{\gamma}}(\phi U)+\left(\mu / \kappa_{1}\right) \widetilde{\nabla}_{\dot{\gamma}} \mathcal{N} \\
& =\left(v / \kappa_{1}\right)\left\{\nabla_{\xi}(\phi U)+g(A \xi, \phi U) \mathcal{N}\right\}-\left(\mu / \kappa_{1}\right) A \xi \\
& =\left(v / \kappa_{1}\right)\left\{-v \xi+\phi \nabla_{\xi} U\right\}-\left(\mu / \kappa_{1}\right)(\mu \xi+v U) \\
& =-\left\{\left(\mu^{2}+v^{2}\right) / \kappa_{1}\right\} \xi+\left(v / \kappa_{1}\right)\left(\phi \nabla_{\xi} U-\mu U\right) .
\end{aligned}
$$

We shall verify $\phi \nabla \xi U=\mu U$. First, it is clear that $\phi \nabla_{\xi} U$ is perpendicular to $\xi$. Secondly, $\phi \nabla_{\xi} U$ is perpendicular to $\phi U$, because

$$
g\left(\phi \nabla_{\xi} U, \phi U\right)=g\left(\nabla_{\xi} U, U\right)-\eta\left(\nabla_{\xi} U\right) \eta(U)=0 .
$$

So we can verify our assertion when $n=2$. Next, when $n \geq 3$, there exists a vector $Y(\neq 0) \in T^{0} M$ satisfying $Y \perp U$ and $Y \perp \phi U$. For such a vector $Y$, we can take a vector $X \in T^{0} M$ with $\phi X=Y$. This $X$ is also perpendicular to $U$ and $\phi U$. Then, owing to (4.3), we find $v g\left(\nabla_{\xi} X, U\right)=0$. Since $v \neq 0$, we have

$$
\begin{aligned}
0 & =g\left(\nabla_{\xi} X, U\right)=-g\left(X, \nabla_{\xi} U\right) \\
& =-g\left(\phi X, \phi \nabla_{\xi} U\right)-\eta(X) \eta\left(\nabla_{\xi} U\right)=-g\left(Y, \phi \nabla_{\xi} U\right) .
\end{aligned}
$$

Accordingly, $\phi \nabla \xi U$ is perpendicular to $Y$. Lastly, under our assumption, (4.5) yields $g\left(U, \phi \nabla_{\xi} U\right)=\mu$. We thus obtain $\phi \nabla_{\xi} U=\mu U$. Consequently, (4.16) implies $\widetilde{\nabla}_{\dot{\gamma}} V_{2}=$ $-\kappa_{1} \dot{\gamma}$ for some constant $\kappa_{1}$. Hence, every integral curve $\gamma$ of the characteristic vector field $\xi$ on $M_{*}$ is a circle in $\widetilde{M}_{n}(c)$.

Let us consider the case that the curve $\gamma$ has a point that is not contained in $M_{*}$. As mentioned above, if the curve $\gamma$, defined on a connected open interval $I=(a, b)$, is contained in $M_{*}$, then the function $v$ is constant along $\gamma$. Since $v$ is continuous on the whole of $M$ and $v(\gamma(s)) \neq 0$ on $I$, we see $v(\gamma(a))$ and $v(\gamma(b))$ cannot vanish and hence the curve $\gamma$ starting at a point $p \in M_{*}$ cannot intersect the subset

$$
M_{0}=\{p \in M \mid v(p)=0\} .
$$

Therefore, we have only to examine an integral curve $\gamma: I \rightarrow M$ of $\xi$ that satisfies $\gamma(s) \in M_{0}$ for all $s \in I$. By the definition of $v$, we have $A \xi_{\gamma(s)}=\mu \xi_{\gamma(s)}$, so that $\widetilde{\nabla}_{\dot{\gamma}} \dot{\gamma}=\nabla_{\xi} \xi+g(A \xi, \xi) \mathcal{N}=\phi A \xi+\mu \mathcal{N}=\mu \mathcal{N}$. Recall that $\mu$ is constant on $M$. If $\mu=0$, $\gamma$ is a geodesic, that is, a circle of null curvature in $\widetilde{M}_{n}(c)$. If $\mu \neq 0$, we find

$$
\widetilde{\nabla}_{\dot{\gamma}} \mathcal{N}=-A \xi=-\mu \xi .
$$

Thus $\gamma$ is a circle of curvature $\kappa_{1}=\mu$. The "only if" part of Theorem 1.1 is proved.

We shall show the converse. Assume that every integral curve of the characteristic vector field $\xi$ on $M$ is a circle in $\widetilde{M}_{n}(c)$. Take an arbitrary point $p$ of $M_{\star}$ and let $\gamma$ be an integral curve of $\xi$ through $p$. We can use (4.12) (4.15). Since $\gamma$ is a circle in $\widetilde{M}_{n}(c)$, the first curvature $\kappa_{1}$ and the holomorphic torsion $\tau_{12}$ of $\gamma$ are constant along $\gamma$ (see Section 2). Hence, (4.13) and (4.15) imply that the functions $\mu$ and $v$ are constant along $\gamma$, so that

$$
\xi \mu=\xi \nu=0 \quad \text { on } M_{\star} .
$$

Moreover, (4.16) is now valid. Noting that $\gamma$ is of proper order 2 and $v \neq 0$, we have

$$
\phi \nabla_{\xi} U=\mu U \text {, }
$$


which, together with (4.1), yields

$$
\nabla_{\xi} U=-\mu \phi U
$$

In fact, we see that

$$
\mu \phi U=\phi^{2} \nabla_{\xi} U=-\nabla_{\xi} U+\eta\left(\nabla_{\xi} U\right) \xi=-\nabla_{\xi} U-g\left(\nabla_{\xi} \xi, U\right) \xi=-\nabla_{\xi} U .
$$

We now derive that the function $\mu$ is constant on $M_{*}$. It follows from (4.6) and (4.17) that

$$
U \mu=0 .
$$

The following is immediate from (4.5) and (4.18):

$$
(\phi U) \mu=0 .
$$

If $n \geq 3$, we may choose a vector $X(\neq 0) \in T^{0} M$ that is orthogonal to $U$ and $\phi U$. For such a vector $X$, by (4.3) and (4.19) one can see that

$$
X \mu=-v g\left(\nabla_{\xi} X, U\right)=v g\left(X, \nabla_{\xi} U\right)=-v g(X, \mu \phi U)=0 .
$$

From (4.17), (4.20), (4.21), and (4.22) we conclude that $\mu$ is constant on $M_{\star}$ as desired. Hence Trace $A$ is constant on an open dense subset $M_{\star}$ and on the whole $M$ by continuity. Therefore, we conclude that the ruled real hypersurface $M$ has constant mean curvature. Theorem 1.1 is now proved.

We next prove Theorem 1.3.

Proof of Theorem 1.3 Let $M$ be a ruled real hypersurface in a nonflat complex space form $\widetilde{M}_{n}(c), n \geq 2$. Suppose that all integral curves of the characteristic vector field $\xi$ on $M$ are strongly congruent with each other by holomorphic isometries of $\widetilde{M}_{n}(c)$.

First of all, we note that any integral curve $\gamma$ of $\xi$ cannot be a geodesic in $\widetilde{M}_{n}(c)$. In fact, if there exists such an integral curve, by our hypothesis all integral curves of $\xi$ on $M$ are geodesics in $\widetilde{M}_{n}(c)$. Then $\widetilde{\nabla}_{\xi} \xi=0$. In particular, its tangential component vanishes so that $\nabla_{\xi} \xi=\phi A \xi=0$. This means the characteristic vector $\xi$ is principal at any point of $M$. However, it is impossible for ruled real hypersurfaces. Hence, the first curvature of each integral curve $\gamma$ of $\xi$ does not vanish and the holomorphic torsion $\tau_{12}$ can be defined.

Next, for arbitrary two points $p, q$ in $M_{*}$ we choose integral curves $\gamma_{1}, \gamma_{2}$ of $\xi$ that are contained in $M_{\star}$ and satisfy $\gamma_{1}(0)=p, \gamma_{2}(0)=q$. Since two curves $\gamma_{1}$ and $\gamma_{2}$ are strongly congruent by holomorphic isometry of $\widetilde{M}_{n}(c)$, from Theorem A we have

$$
\kappa_{1}^{\gamma_{1}}(s)=\kappa_{1}^{\gamma_{2}}(s), \quad \tau_{12}^{\gamma_{1}}(0)=\tau_{12}^{\gamma_{2}}(0) .
$$

These, together with (4.13) and (4.15), yield

$$
\begin{gathered}
v\left(\gamma_{1}(s)\right)^{2}+\mu\left(\gamma_{1}(s)\right)^{2}=v\left(\gamma_{2}(s)\right)^{2}+\mu\left(\gamma_{2}(s)\right)^{2}, \\
\mu\left(\gamma_{1}(0)\right) / \kappa_{1}^{\gamma_{1}}(0)=\mu\left(\gamma_{2}(0)\right) / \kappa_{1}^{\gamma_{2}}(0) .
\end{gathered}
$$

Hence, we obtain $\mu(p)=\mu(q), v(p)=v(q)$ for any $p, q \in M_{*}$. Since the subset $M_{*}$ is open and dense in $M$, the functions $\mu$ and $v$ are constant on $M$. On the other hand, substituting $\phi U$ for $X$ in (4.4) we get $(\phi U) v=v^{2}+(c / 4)$. Suppose that $c>0$. We then have $(\phi U) v>0$ from the above. But it contradicts the fact that $v$ is constant. 
Therefore, the ambient space must be a complex hyperbolic space $\mathbb{C} H^{n}(c)$. The constancy of $\mu$ and $v$ implies that $M$ has constant principal curvatures (see Section 3), and accordingly, by virtue of Theorem B, we obtain the conclusion of our Theorem 1.3.

It remains to prove Corollary 1.4. Suppose that all integral curves of the characteristic vector field $\xi$ on a ruled real hypersurface $M$ are circles of the same curvature in $\widetilde{M}_{n}(c)$. By Corollary 1.2 , one finds that the real hypersurface $M$ is minimal in $\widetilde{M}_{n}(c)$. Hence every integral curve of $\xi$ on $M$ is a totally real circle and its holomorphic torsion $\tau_{12}$ vanishes. Then, in view of Theorem A and Theorem 1.3, our Corollary 1.4 follows.

The following problem related to our Theorem 1.3 is still open.

Problem Let $M$ be a ruled real hypersurface in a complex hyperbolic space $\mathbb{C} H^{n}(c)$, $n \geq 2$. If all integral curves of the characteristic vector field $\xi$ on $M$ are congruent in the usual sense with each other by holomorphic isometries of $\mathbb{C} H^{n}(c)$, is $M$ homogeneous in this ambient space?

Acknowledgements The authors would like to express their appreciation to the referee for his or her useful suggestions.

\section{References}

[1] T. Adachi, T. Bao, and S. Maeda, Congruence classes of minimal ruled real hypersurfaces in a nonflat complex space form. Hokkaido Math. J. 43(2014), 137-150. http://dx.doi.org/10.14492/hokmj/1392906097

[2] T. Adachi and S. Maeda, Global behaviours of circles in a complex hyperbolic space. Tsukuba J. Math. 21(1997), 29-42. http://dx.doi.org/10.21099/tkbjm/1496163159

[3] T. Adachi, S. Maeda, and S. Udagawa, Circles in a complex projective space. Osaka J. Math. 32(1995), 709-719.

[4] J. Berndt and H. Tamaru, Cohomogeneity one actions on noncompact symmetric spaces of rank one. Trans. Amer. Math. Soc. 359(2007), 3425-3438. http://dx.doi.org/10.1090/S0002-9947-07-04305-X

[5] M. Domínguez-Vázquez and O. Pérez-Barral, Ruled hypersurfaces with constant mean curvature in complex space forms. arxiv:1802.09341

[6] M. Lohnherr and H. Reckziegel, On ruled real hypersurfaces in complex space forms. Geom. Dedicata 79(1999), 267-286. http://dx.doi.org/10.1023/A:1005000122427

[7] S. Maeda and Y. Ohnita, Helical geodesic immersions into complex space forms. Geom. Dedicata 30(1989), 93-114. http://dx.doi.org/10.1007/BF02424315

[8] R. Niebergall and P. J. Ryan, Real hypersurfaces in complex space forms. In: Tight and taut submanifolds. Cambridge University Press, Cambridge, 1998, pp. 233-305.

[9] R. Takagi, On homogeneous real hypersurfaces in a complex projective space. Osaka J. Math. 10(1973), 495-506.

[10] - Real hypersurfaces in a complex projective space with constant principal curvatures. II. J. Math. Soc. Japan 27(1975), 507-516. http://dx.doi.org/10.2969/jmsj/02740507

Department of Mathematics, Saga University, 1 Honzyo, Saga 840-8502, Japan

Email: sayaki@cc.saga-u.ac.jp

Department of Science, National Institute of Technology, Matsue College, Matsue, Shimane 690-8518, Japan

Email: h-tanabe@matsue-ct.jp

Department of Mathematics, School of Medicine, Nihon University, Itabashi, Tokyo 173-0032, Japan

Email: udagawa.seiichi@nihon-u.ac.jp 